07

\title{
Экспериментальная и теоретическая оценка рентгеноконтрастности тонкомерных образцов никелида титана с микронными покрытиями на основе Ті и Та
}

\author{
(C) Е.Ю. Гудимова, ${ }^{1,2}$ Л.Л. Мейснер, ${ }^{1,2}$ С.Н. Мейснер, ${ }^{1}$ О.И. Шабалина, ${ }^{1,2}$ А.А. Бощенко, ${ }^{3}$ А.Е. Баев, ${ }^{3}$ \\ С.И. Винтизенко ${ }^{3}$ \\ ${ }^{1}$ Институт фризики прочности и материаловедения СО РАН, \\ 634055 Томск, Россия \\ ${ }^{2}$ Национальный исследовательский Томский государственный университет, \\ 634050 Томск, Россия \\ ${ }^{3}$ Томский национальный исследовательский медицинский центр РАН, Научно-исследовательский институт кардиологии, \\ 634012 Томск, Россия \\ e-mail: egu@ispms.tsc.ru
}

Поступило в Редакцию 1 мая 2019 г.

В окончательной редакции 1 мая 2019 г.

Принято к публикации 21 октября 2019 г.

\begin{abstract}
Проанализирована перспектива использования покрытий микронной толщины, содержащих тантал, для эффективного повышения рентгеноконтрастности миниатюрных металлических изделий, используемых в эндохирургии. Исследованы свойства рентгеноконтрастности покрытий из Та и Тi-Та толщиной $1-2 \mu \mathrm{m}$, сформированных на поверхностях образцов проволоки из TiNi-сплава диаметром $152 \mu \mathrm{m}$. Приведены сравнительные данные расчетных (по значениям массового коэффициента ослабления рентгеновских лучей) и экспериментальных, полученных рентгенологическим методом на ангиографической установке, характеристик рентгеноконтрастности материалов покрытий. Показано, что наличие $\mathrm{Ti}_{60-70} \mathrm{Ta}_{40-30}$-покрытий толщиной $1-2 \mu \mathrm{m}$ на поверхности проволоки из TiNi-сплава приводит к увеличению рентгеноконтрастности материала в 2 раза. Проанализировано изменение контрастности образцов TiNi в зависимости от концентрации тантала в покрытии при применении основных ангиографических режимов съемки.
\end{abstract}

Ключевые слова: сплавы с эффектом памяти формы, никелид титана, покрытия на основе Ті и Та, рентгеноконтрастность, сердечно-сосудистая хирургия.

DOI: $10.21883 /$ JTF.2020.04.49094.184-19

\section{Введение}

За последние годы в диагностике и лечении сердечнососудистых заболеваний достигнуты значительные успехи. Широкое применение малоинвазивных методов в кардиохирургии позволило свести к минимуму степень травмирования биологических тканей пациента в процессе оперативного вмешательства. Однако для осуществления строгого контроля за перемещением, положением и ориентацией устанавливаемых имплантатов (стенты, окклюдеры и др.) во время и после эндоваскулярных операций необходима четкая визуализация изделия под слабым рентгеновским излучением.

Сплавы на основе никелида титана (TiNi) являются перспективными материалами медицинского назначения благодаря своим уникальным функциональным свойствам, таким как эффекты памяти формы и сверхэластичности. Однако данные сплавы обладают низким коэффициентом поглощения рентгеновских лучей. Увеличение рентгеноконтрастности TiNi-сплавов возможно в результате осаждения на его поверхность покрытия из рентгеноконтрастного материала, например, тантала [1-3]. Как известно [4-6], тантал успешно используется в качестве медицинского материала, в том чис- ле и для изготовления миниатюрных имплантатов для размещения в тонкие кровеносные сосуды. Он обладает бо́льшим коэффициентом поглощения рентгеновских лучей $\left(\mu_{\mathrm{m}}(\mathrm{Ta})=7.59 \mathrm{~cm}^{2} / \mathrm{g}\right.$ при $\left.80 \mathrm{keV}[7]\right)$, по сравнению c TiNi-сплавом или чистым титаном. Оксидные пленки на основе тантала являются коррозионно-стойкими и инертными к биологическим средам (жидкостям и тканям) [5]. Как было показано [1-3], наличие покрытия из тантала толщиной $3-5 \mu \mathrm{m}$ на поверхности TiNi-сплава позволило не только увеличить рентгеноконтрастность материала, но и подавить выход ионов $\mathrm{Ni}$, повысить параметры гемосовместимости и пролиферативную активность эндотелиальных клеток на поверхности образцов с покрытиями. Основным недостатком таких покрытий является большое различие температурных коэффициентов линейного расширения $\alpha$ и модуля упругости $E$ тантала $\left(\alpha(\mathrm{Ta})=6.6 \cdot 10^{-6} \mathrm{~K}^{-1}\right.$, $E(\mathrm{Ta})=190 \mathrm{GPa})[8]$ и TiNi-сплава в аустенитном состоянии $\left(\alpha(\mathrm{TiNi})=11 \cdot 10^{-6} \mathrm{~K}^{-1}, E(\mathrm{TiNi})=75 \mathrm{GPa}\right)[9]$. В связи с этим существует высокий риск отслоения покрытия из Та от материала подложки из TiNi-сплава из-за низкой адгезионной прочности его сцепления с подложкой. 
Решением проблемы повышения адгезионной прочности покрытия [10] является выбор в качестве материала покрытия сплава с механическими и деформационными характеристиками, близкими характеристикам материала подложки - TiNi-сплава. Так, сплавы $\mathrm{Ti}_{60-70} \mathrm{Ta}_{40-30}$ (at.\%) обладают высокотемпературным эффектом памяти формы, температурный интервал мартенситного превращения этих сплавов на $50-100^{\circ} \mathrm{C}$ выше комнатной температуры [11] и при температуре человеческого тела данные сплавы находятся в высокоэластичном мартенситном состоянии. Согласно [12], модуль упругости сплава $\mathrm{Ti}_{65} \mathrm{Ta}_{35}$ (at.\%) $(E \approx 70 \mathrm{GPa})$ близок по величине к значению $E$ для TiNi-сплава и сопоставим с упругими характеристиками мышечных тканей. В работах $[13,14]$ было показано, что данные свойства сохраняются и в тонкопленочных Ті-Та-покрытиях (толщина 200-300 nm) того же состава, сформированных в результате одновременного магнетронного распыления Ті- и Та-мишеней. Таким образом, сочетание близких механических свойств $\mathrm{Ti}_{60-70} \mathrm{Ta}_{40-30}$ и TiNi-сплавов и высокой рентгенопоглощательной способности $\mathrm{Ti}_{60-70} \mathrm{Ta}_{40-30}$-сплавов позволило бы эффективно повысить рентгеноконтрастность композиционной системы „,Ti $60-70 \mathrm{Ta}_{40-30}-$ покрытие $/ \mathrm{TiNi}-$ подложка“ при толщине покрытия, не превышающей нескольких микрометров.

Цель работы - разработка способа предварительной оценки уровня рентгеноконтрастности материала на основе сравнения теоретически рассчитанных и экспериментально (рентгенологически) измеренных параметров контрастности и оценка эффективности повышения рентгеноконтрастности миниатюрных металлических изделий для эндохирургии с использованием покрытий микронной толщины, содержащих тантал, на примере тонкомерных (проволока диаметром $150 \mu \mathrm{m}$ ) образцов никелида титана.

\section{1. Материал и методики эксперимента}

Образцы для исследования были изготовлены из проволоки TiNi-сплава марки TH1 (OOO „Промышленный центр МАТЭК-СПФ“). Химический состав сплава (wt.\%): $55.54 \mathrm{Ni}, 0.05 \mathrm{C}, 0.045 \mathrm{O}, 0.009 \mathrm{~N}$, баланс Ti. Температура конца обратного мартенситного превращения $A_{\mathrm{K}}=304 \mathrm{~K}$. Диаметр проволоки в состоянии поставки, измеренный по РЭМ-изображениям, $152 \mu \mathrm{m}$.

Модификацию поверхности образцов осуществляли на установке „РИТМ-СП“ в ИСЭ СО РАН (г. Томск). Предварительную электронно-пучковую обработку с целью очистки проводили в режиме импульсного плавления: ускоряющее напряжение $U=25 \mathrm{kV}$, число импульсов $n=32$ (такие образцы далее обозначены, как $\mathrm{TiNi}^{\mathrm{EB}}$ ). Покрытия Ti-Tа получали путем магнетронного распыления мишеней из титана и тантала и одновременного соосаждения этих элементов на поверхности равномерно вращающихся образцов TiNi-проволоки.
Таблица 1. Режимы и параметры съемки, используемые при ангиографических исследованиях

\begin{tabular}{c|c|c|c|c|c|c}
\hline \multirow{2}{*}{$\begin{array}{c}\text { № } \\
\text { п/п }\end{array}$} & Режим & \multicolumn{5}{|c}{ Параметры съемки } \\
\cline { 3 - 6 } & $U, \mathrm{kV}$ & $I, \mathrm{~mA}$ & $t, \mu \mathrm{s}$ & $\begin{array}{c}\text { С-фильтр, } \\
\text { тm }\end{array}$ & $\begin{array}{c}\text { Допол- } \\
\text { нительное } \\
\text { излучение }\end{array}$ \\
\hline 1 & Care & 54 & 12 & 3.7 & 0.2 & \multirow{2}{*}{-} \\
\hline 2 & xCare & 55 & 13 & 3.6 & 0.3 & \\
\hline 3 & xхCare & 60 & 13 & 3.6 & 0.6 & \multirow{2}{*}{ включено } \\
\hline 4 & Coro & 50 & 13 & 3.5 & 0.1 &
\end{tabular}

Режимы обработок: (1) $I(\mathrm{Ta})=0.5 \mathrm{~A}, I(\mathrm{Ti})=1.54 \mathrm{~A}$, $U(\mathrm{Ta})=(303 \pm 2) \mathrm{V}, U(\mathrm{Ti})=(344 \pm 1) \mathrm{V}, t=1400 \mathrm{~s}$ для формирования покрытия состава $\mathrm{Ti}_{70} \mathrm{Ta}_{30}$ (at.\%) и толщиной $1 \mu \mathrm{m} ; \quad(2) \quad I(\mathrm{Ta})=0.5 \mathrm{~A}, \quad I(\mathrm{Ti})=1.16 \mathrm{~A}$, $U(\mathrm{Ta})=(302 \pm 3) \mathrm{V}, U(\mathrm{Ti})=(331 \pm 2) \mathrm{V}, t=1400 \mathrm{~s}-$ покрытие состава $\mathrm{Ti}_{60} \mathrm{Ta}_{40}($ at.\%) и толщиной $2 \mu \mathrm{m}$; (3) $I(\mathrm{Ta})=1.5 \mathrm{~A}, U(\mathrm{Ta})=328 \pm 1 \mathrm{~V}, t=1200 \mathrm{~s}-$ покрытие из чистого тантала толщиной $2 \mu \mathrm{m}$.

Для сравнения в настоящей работе были исследованы проволочные образцы TiNi-сплава с поверхностью, очистку которой проводили без привлечения электронно-пучковой обработки. Образцы последовательно подвергали химическому травлению в растворе кислот $\left(3 \mathrm{~h} \mathrm{HNO}_{3}+1 \mathrm{~h} \mathrm{HF}\right)$, электролитической полировке в охлажденном до $T=273 \mathrm{~K}$ растворе кислот $\left(3 \mathrm{~h} \mathrm{CH} 3 \mathrm{COOH}+1 \mathrm{~h} \mathrm{HClO}_{4}\right)$ и 3 -х-кратной промывке дистиллированной водой в ультразвуковой ванне.

Контроль сплошности и измерение элементного состава покрытий проводили на растровом электронном микроскопе (РЭМ) TESCAN VEGA II LMU (TESCAN Brno s.r.o., Чехия) с использованием системы рентгеновского энергодисперсионного микроанализа Oxford Instruments (Великобритания) Oxford INCA Energy350 при ускоряющем напряжении $10 \mathrm{kV}$ (ЦКП „Нанотех“, ИФПМ СО РАН, г. Томск). Погрешности определения элементного состава: $\mathrm{Ti}-2$ at.\%, $\mathrm{Ni}-2$ at.\%, Ta -1 at. $\%, \mathrm{O}-4$ at.\%, C -5 at. $\%$.

Теоретическая оценка рентгеноконтрастности материала заключалась в расчете массового коэффициента ослабления рентгеновских лучей для двойных систем Ti-Tа и Ti-Ni по формуле

$$
\mu_{\mathrm{m}}(\mathrm{Te}-\mathrm{Me})=\mu_{\mathrm{m}}(\mathrm{Ti})+\mu_{\mathrm{m}}(\mathrm{Me}) \cdot w(\mathrm{Me})
$$

где $w$ - концентрация элемента в весовых процентах; $\mathrm{Me:} \mathrm{Ta,} \mathrm{Ni.} \mathrm{Для} \mathrm{расчета} \mathrm{использовали} \mathrm{значения} \mathrm{массовых}$ коэффициентов чистых элементов $\mu_{\mathrm{m}}(\mathrm{Ti})=0.41 \mathrm{~cm}^{2} / \mathrm{g}$, $\mu_{\mathrm{m}}(\mathrm{Ta})=7.59 \mathrm{~cm}^{2} / \mathrm{g}, \mu_{\mathrm{m}}(\mathrm{Ni})=0.73 \mathrm{~cm}^{2} / \mathrm{g}$, полученных при энергии рентгеновских фотонов $80 \mathrm{keV}$ [7], что соответствует энергии рентгеновского пучка, используемого при ангиографических операциях. 


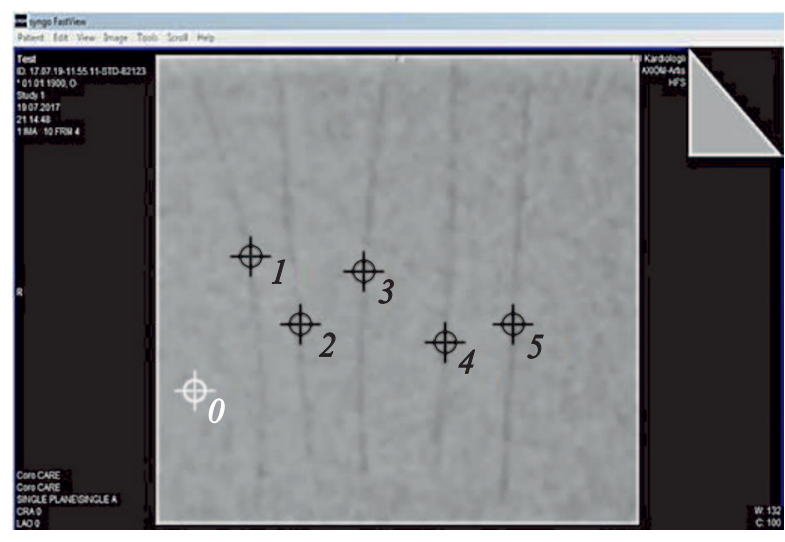

Рис. 1. Пример ангиографического изображения образцов проволоки из TiNi-сплава, режим „Care“.

Экспериментальную оценку рентгеноконтрастности сплава TiNi до и после осаждения покрытий осуществляли на базе отделения рентгенохирургических методов диагностики и лечения Научно-исследовательского института кардиологии Томского национального исследовательского медицинского центра (г. Томск). Исследование выполняли с помощью ангиографической установки Axiom Artis dFC (Siemens Health Care $\mathrm{GmbH}$, Германия) во время процедуры стентирования магистральных коронарных артерий, размещая проволочные образцы TiNi-сплава в проекции сердца под лежащим на спине пациентом. Все процедуры, выполненные в исследовании с участием людей, проведены в соответствии с принципами Хельсинкской декларации 1964 г. и ее последующим изменениям и одобрены комитетом по биомедицинской этике НИИ кардиологии Томского НИМЦ. От каждого из включенных в исследование участников было получено добровольное информированное согласие. Позиционирование и фокусировку проводили в режиме импульсной флюороскопии, а регистрацию изображений - в режиме цифровой субтракционной ангиографии с серийной съемкой 10-20 кадров в секунду. Было выполнено 4 серии экспериментов в стандартных низкодозовых режимах („Care“, „хCare“, „хxСare“ и „Соro“), характеризующихся разными основными характеристиками съемки: напряжения $(U)$ и силы тока $(I)$, подаваемых на трубку, времени экспозиции $(t)$,

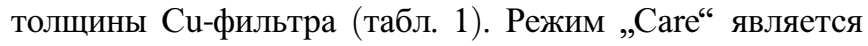
наиболее интенсивным и применяется преимущественно для пациентов с избыточной массой тела. Режим „хxСare“ характеризуется минимальной дозой облучения, что позволяет его использовать для пациентов с низкой массой тела, детей и подростков. Режим „хСаre“ является промежуточным между двумя предельными режимами, описанными выше, и применяется для пациентов с массой тела $50-90 \mathrm{~kg}$. В работе также проводились исследования в режиме „Coro“, который включает в себя дополнительное излучение, основанное на эффекте флуоресценции.
Таблица 2. Значения удельной массы тантала в покрытиях, осажденных на поверхности TiNi сплава

\begin{tabular}{c|c|c}
\hline Образец & Толщина покрытия, $\mu \mathrm{m}$ & $\begin{array}{c}\text { Удельная масса } \\
\text { тантала } m_{\mathrm{Ta}}, 10^{-10} \mathrm{~g}\end{array}$ \\
\hline $\mathrm{Ti}_{70} \mathrm{Ta}_{30} / \mathrm{TiNi}$ & 1 & 47.43 \\
$\mathrm{Ti}_{60} \mathrm{Ta}_{40} / \mathrm{TiNi}$ & 2 & 109.19 \\
$\mathrm{Ta} / \mathrm{TiNi}$ & 2 & 152.5
\end{tabular}

Для набора статистических данных в каждом режиме проводили съемку 15 кадров. На каждом кадре измеряли по 5 значений в наиболее контрастных местах исследуемого проволочного образца (рис. 1). Обработку изображений для получения численных значений коэффициента рентгеноконтрастности $(K)$ проводили с помощью программы AdobePhotoshop 10 в градиенте серого по шкале RGB от 0 (черный) до 255 (белый) согласно [15]. Коэффициент $K$ рассчитывали по формуле

$$
K=\left[\frac{R G B_{\text {background }}-R G B_{i}}{R G B_{\text {background }}}\right] \times 100 \%,
$$

где $i$ - номер образца.

В связи с тем, что в настоящей работе варьировалась толщина и состав покрытий, для выявления корреляционных зависимостей был проведен расчет удельной массы тантала в осажденных покрытиях. Считали, что покрытие представляет собой цилиндрическую трубку толщиной 1 или $2 \mu \mathrm{m}$ и высотой $1 \mu \mathrm{m}$. Удельную массу тантала в объеме такой цилиндрической трубки находили по формуле

$$
m_{\mathrm{Ta}}=V_{\mathrm{Ti}-\mathrm{Ta}} \cdot w_{\mathrm{Ta}} \cdot \rho_{\mathrm{Ta}},
$$

где $\rho_{\mathrm{Ta}}-$ плотность тантала, $w_{\mathrm{Ta}}-$ концентрация тантала в весовых процентах, $V_{\mathrm{Ti}-\mathrm{Ta}}$ - объем Ті-Та-покрытия. Результаты расчета приведены в табл. 2.

\section{2. Результаты и их обсуждение}

\section{1. Морфология и элементный состав проволочных образцов}

В состоянии поставки поверхность проволоки TiNi-сплава неоднородна (рис. 2, a). Анализ элементного состава, согласно данным энергодисперсионной спектрометрии, полученным с площади $100 \times 100 \mu \mathrm{m}$, показал, что в поверхностном слое толщиной $\sim 1 \mu \mathrm{m}$ содержится высокая концентрация углерода 82 at.\% (табл. 3). Это обусловлено процессом получения проволоки, а именно ее многократной протяжкой через фильеры с использованием графитовой смазки для достижения необходимого диаметра.

После электрохимической очистки поверхность проволоки приобрела развитый рельеф (рис. 2,b), что 


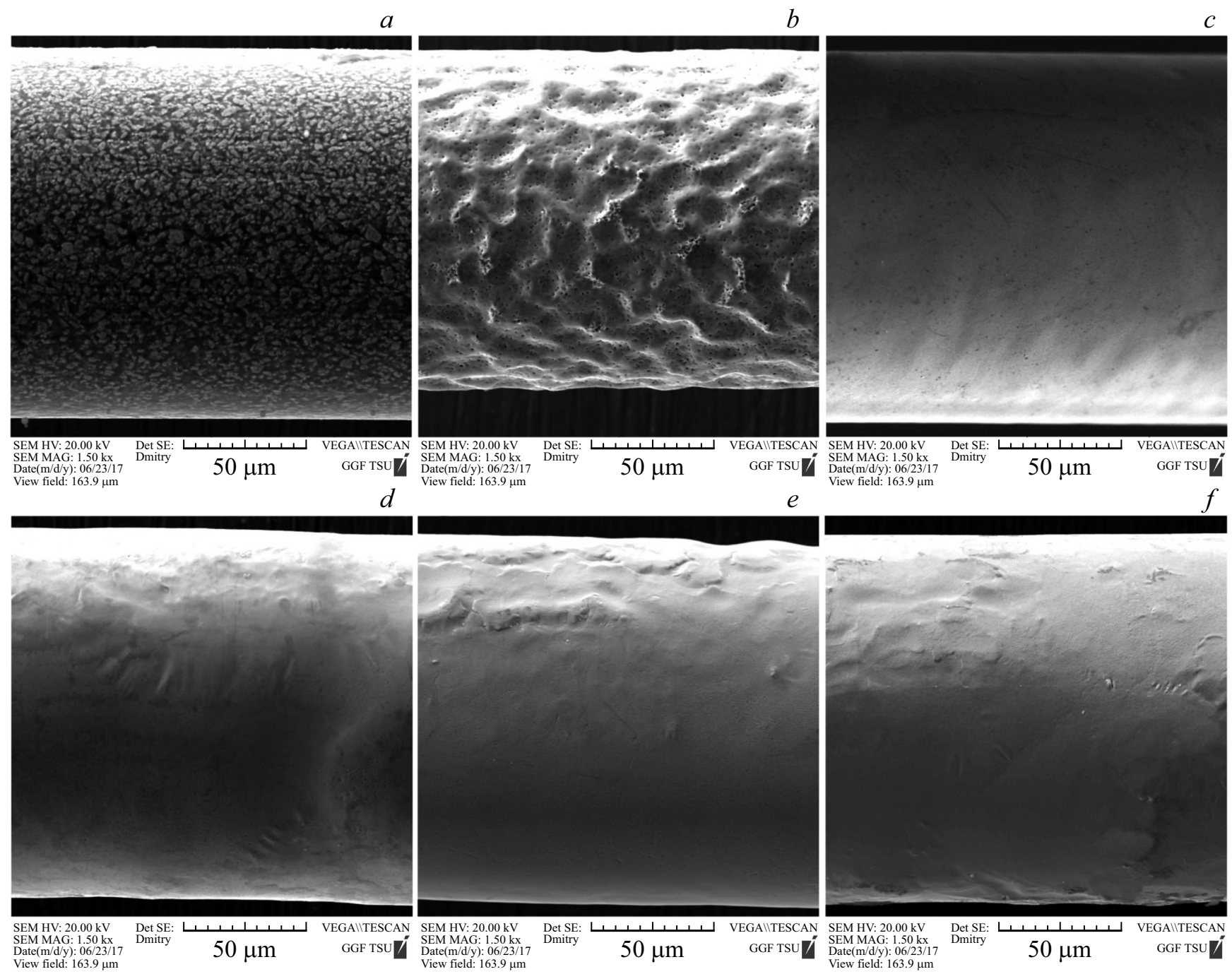

Рис. 2. РЭМ-изображения поверхности образцов проволоки из TiNi-сплава: исходная поверхность $(a)$, после электрохимической обработки $(b)$, после электронно-пучковой обработки $\mathrm{TiNi}^{\mathrm{EB}}(c)$, с покрытиями - $\mathrm{Ti}_{70} \mathrm{Ta}_{30} / \mathrm{TiNi}(d), \mathrm{Ti}_{60} \mathrm{Ta}_{40} / \mathrm{TiNi}_{(}(e), \mathrm{Ta} / \mathrm{TiNi}(f)$.

привело к размытию ее границ на изображении и формированию большого количества дефектов. Кроме того, диаметр проволоки уменьшился на $\sim 10 \mu \mathrm{m}$. Следует отметить, что в результате данной обработки наличие кислорода и углерода в поверхностной области не обнаружено (табл. 3).

Как видно из рис. 2, $c$, электронно-пучковая обработка образцов в режиме импульсного плавления привела к выглаживанию и формированию однородной поверхности образцов по сравнению с их исходным состоянием. На РЭМ-изображении границы проволоки ровные и четкие. Диаметр образцов относительно исходного размера не изменился и составил $152 \mu \mathrm{m}$. Анализ элементного состава показал отсутствие углерода и наличие кислорода в концентрации 17 at.\%. На основании данных результатов далее в качестве предварительной обработки поверхности проволочных образцов перед нанесением покрытий применяли электронно-пучковую обработку.
Таблица 3. ЭДС/РЭМ $(U=10 \mathrm{kV})$ данные о среднем элементном составе поверхностных слоев толщиной $\sim 1 \mu \mathrm{m}$ в TiNi образцах без покрытий и с покрытиями

\begin{tabular}{l|c|c|c|c|c|c}
\hline Образец & $\begin{array}{c}\mathrm{Ti}, \\
\text { at.\% }\end{array}$ & $\begin{array}{c}\mathrm{Ta}, \\
\text { at.\% }\end{array}$ & $\begin{array}{c}\mathrm{Ni}, \\
\text { at.\% }\end{array}$ & $\begin{array}{c}\mathrm{O}, \\
\text { at.\% }\end{array}$ & $\begin{array}{c}\mathrm{C}, \\
\text { at.\% }\end{array}$ & Ti : Ta $: \mathrm{Ni}$ \\
\hline $\mathrm{TiNi}($ поставка) & 5 & - & 5 & 8 & 82 & - \\
$\mathrm{TiNi}$ & 45 & - & 55 & - & - & - \\
$\mathrm{TiNi}^{\mathrm{EB}}$ & 46 & - & 37 & 17 & - & - \\
$\mathrm{Ti}_{70} \mathrm{Ta}_{30} / \mathrm{TiNi}$ & 60 & 18 & 4 & 18 & & $73: 22: 5$ \\
$\mathrm{Ti}_{60} \mathrm{Ta}_{40} / \mathrm{TiNi}$ & 60 & 22 & 3 & 15 & - & $71: 26: 3$ \\
$\mathrm{Ta} / \mathrm{TiNi}$ & 3 & 74 & 6 & 17 & - & $4: 89: 7$
\end{tabular}

Использование метода магнетронного осаждения позволило сформировать морфологически однородные покрытия на основе Ті-Та и Та, равномерно распределенные по всей площади цилиндрической поверхности 


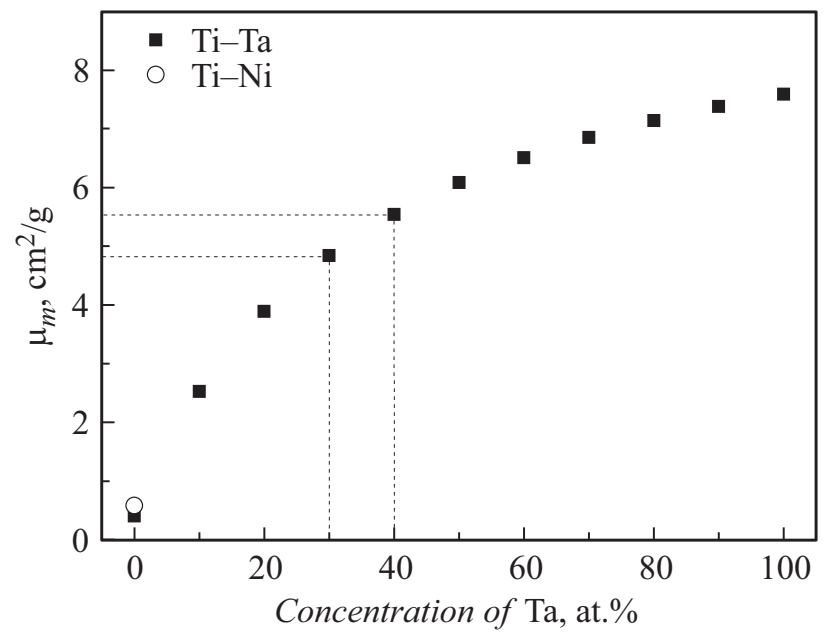

Рис. 3. Зависимость массового коэффициента ослабления рентгеновских лучей $\left(\mu_{\mathrm{m}}\right)$ от концентрации тантала в системе Ti-Ta.

образца без трещин и сколов (рис. $2, d-f)$. Согласно данным энергодисперсионной спектрометрии, соотношение титана и тантала в осажденных покрытиях близко к заданному составу (табл. 3). Небольшая концентрация никеля, фиксируемая данным методом $(U=10 \mathrm{kV})$, обусловлена тем, что анализируемый слой, кроме материала покрытия, включает материал подложки из TiNi.

\section{2. Анализ изменения массового коэффициента ослабления рентгеновских лучей}

Значение массового коэффициента ослабления рентгеновских лучей, рассчитанного в настоящей работе по формуле (1), для TiNi-сплава составляет $\mu_{\mathrm{m}}(\mathrm{TiNi})=0.59 \mathrm{~cm}^{2} / \mathrm{g}$, что в 13 раз ниже значения для чистого тантала $\left(\mu_{\mathrm{m}}(\mathrm{Ta})=7.59 \mathrm{~cm}^{2} / \mathrm{g}\right)$. Как видно из рис. 3 , зависимость коэффициента $\mu_{\mathrm{m}}$ от концентрации тантала в системе Ті-Та имеет нелинейный характер. Увеличение концентрации тантала до 30-40 at.\% приводит к быстрому росту коэффициента $\mu_{\mathrm{m}}$ до $\mu_{\mathrm{m}}\left(\mathrm{Ti}_{60} \mathrm{Ta}_{40}\right)=5.55 \mathrm{~cm}^{2} / \mathrm{g}$. Дальнейшее увеличение концентрации тантала не приводит к значительному изменению коэффициента $\mu_{\mathrm{m}}$. Иными словами, концентрации тантала 30-40 at.\% в двухкомпонентном материале из Тi и Та будет достаточно для увеличения коэффициента $\mu_{\mathrm{m}}$ и соответственно рентгеноконтрастности материала в 10 раз по сравнению с рентгеноконтрастностью TiNi-сплава.

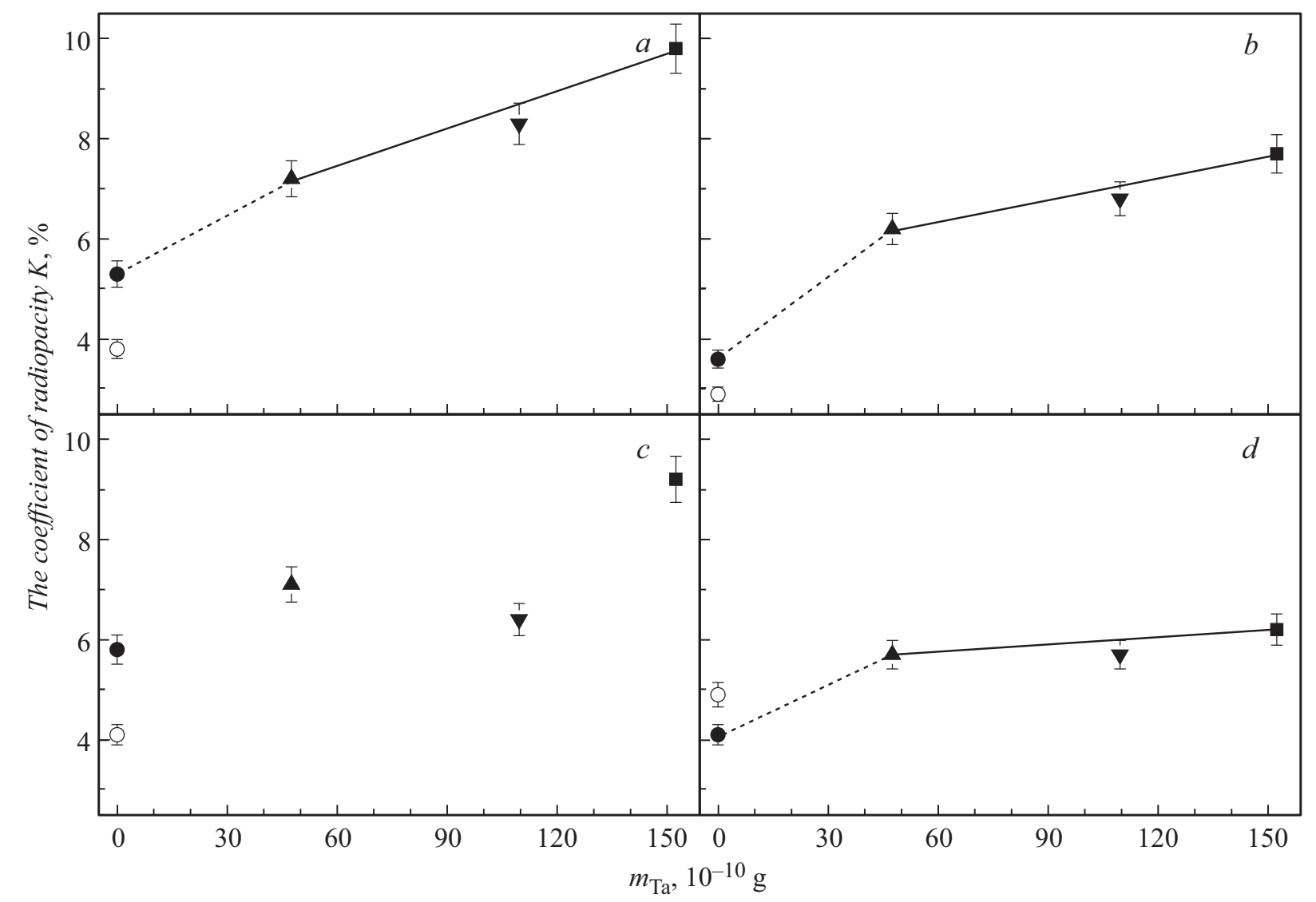

Рис. 4. Зависимости коэффициента рентгеноконтрастности $K$ от удельной массы тантала в покрытии, полученные при различных условиях рентгеновских (ангиографических) съемок в режимах: „Сare“ $(a)$, „хСare“ $(b)$, „ххСаre“ $(c)$, „Соro“ $(d)$. Обозначение образцов: ॰ - TiNi, • - $\mathrm{TiNi}^{\mathrm{EB}}, \boldsymbol{\Delta}-\mathrm{Ti}_{70} \mathrm{Ta}_{30} / \mathrm{TiNi}, \boldsymbol{\nabla}-\mathrm{Ti}_{60} \mathrm{Ta}_{40} / \mathrm{TiNi}, \mathbf{\square}-\mathrm{Ta} / \mathrm{TiNi}$. 


\section{3. Ангиографическая оценка рентгеноконтрастности материала и оценка оптимальных условий визуализации на примере проволочных образцов TiNi-имплантатов}

Ангиографические исследования показали, что независимо от режима съемки значения коэффициента рентгеноконтрастности образцов $\mathrm{TiNi}^{\mathrm{EB}}$ (после электроннопучковой обработки) выше, чем эти значения у образцов с поверхностями, очищенными электрохимическим способом (рис. 4). Повышение рентгеноконтрастности образцов $\mathrm{TiNi}^{\mathrm{EB}}$ обусловлено, в первую очередь, сохранением исходного диаметра проволоки. Кроме того, границы проволоки являются четкими и ровными, что способствует лучшей визуализации и более точному определению контрастности при анализе ангиографических снимков. Осаждение Ті-Та-покрытий толщиной $1-2 \mu \mathrm{m}$ приводит к увеличению коэффициента рентгеноконтрастности в 2 раза относительно его значения для TiNi-cплава. Например, в режиме ,xCare“ $\mathrm{K}\left(\mathrm{Ti}_{70} \mathrm{Ta}_{30} / \mathrm{TiNi}\right)=6 \%, K\left(\mathrm{Ti}_{60} \mathrm{Ta}_{40} / \mathrm{TiNi}\right)=7 \%$, в то время как $K(\mathrm{TiNi})=3 \%$. Следует отметить, что осаждение покрытия из чистого тантала толщиной $2 \mu \mathrm{m}$ повышает рентгеноконтрастность проволоки TiNi сплава в 2.5 раза: $K(\mathrm{Ta} / \mathrm{TiNi})=7.7 \%$ в режиме „хCare“.

Анализ зависимостей $K\left(m_{\mathrm{Ta}}\right)$ показал, что в режимах „Сare“ (рис. 4, $a$ ) и „хСаre“ (рис. 4,b) значения коэффициентов рентгеноконтрастности $K$ линейно увеличились с повышением содержания тантала в покрытиях. Данный характер изменения коэффициента $K$ позволяет прогнозировать увеличение или уменьшение рентгеноконтрастности материала и давать рекомендации по выбору состава покрытия для конкретного режима съемки. При съемке в режиме „ххСаre“ (рис. 4, c) изменение коэффициентов рентгеноконтрастности $K$ имеет хаотичный характер. Большой разброс значений коэффициентов $K$, вероятнее всего, связан с минимальной интенсивностью рентгеновского излучения, которая не позволяет достоверно определить размеры образца на ангиографическом снимке. При использовании режима „Сoro“ (рис. 4, $d$ ) контрастность образцов практически не зависит от толщины и состава покрытий. Изменение значений коэффициента $K$ незначительно и варьируется в пределах ошибки измерений.

Наконец, в настоящей работе были проведены рентгеновские исследования образцов, изготовленных в виде плетеных стентов из той же TiNi-проволоки, использованной в описанных ранее экспериментах. Покрытия, анализируемые в настоящей работе, были нанесены на готовые полуфабрикаты. Образцы-прототипы стента были расположены на операционном столе (под пациентом) таким образом, чтобы на рентгеновском снимке проекции их положения совпали (или были близки) с проекцией стента, имплантируемого в сосуд пациента (указан стрелкой) (рис. 5). Как видно, образцыпрототипы хорошо видны, несмотря на то, что преграда

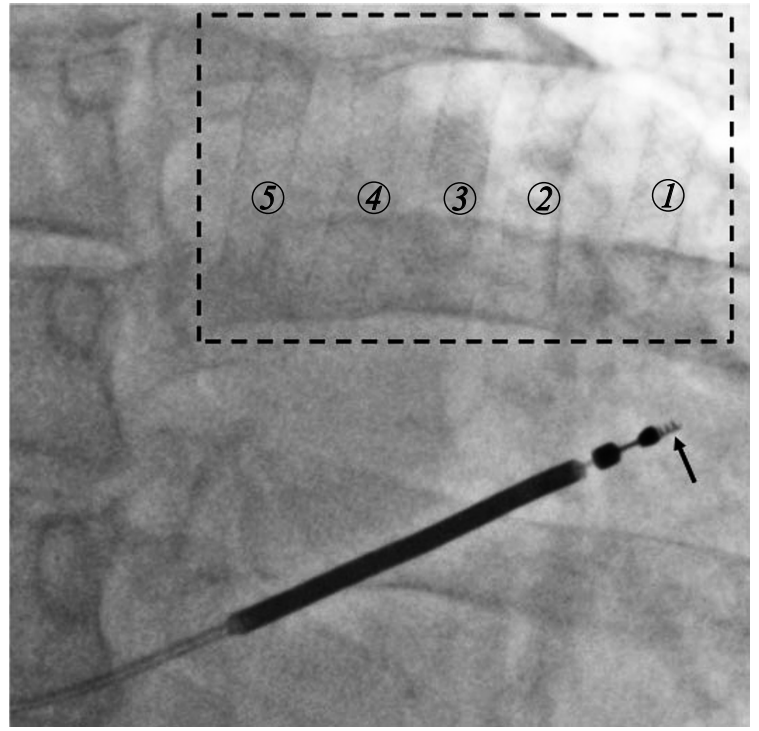

Рис. 5. Рентгеновский (ангиографический, режим „хСаre“) снимок образцов-прототипов плетеных стентов из TiNi проволоки $(\varnothing 152 \mu \mathrm{m})$ и стента (указан стрелкой), имплантируемого в сосуд пациента. Область с изображениями образцов выделена рамкой. Обозначение образцов: $1-\mathrm{TiNi}, 2-\mathrm{TiNi}^{\mathrm{EB}}$, $3-\mathrm{Ti}_{70} \mathrm{Ta}_{30} / \mathrm{TiNi}, 4-\mathrm{Ti}_{60} \mathrm{Ta}_{40} / \mathrm{TiNi}, 5-\mathrm{Ta} / \mathrm{TiNi}$.

на пути распространения рентгеновского пучка до образцов больше, как минимум, в 2 раза, чем до имплантата внутри организма. При этом наиболее четкие изображения имеют именно образцы с микронными покрытиями, содержащими тантал.

\section{Заключение}

Таким образом, результаты проведенных исследований позволяют сделать важное, практически значимое заключение: (i) для удвоенного повышения рентгеноконтрастности тонкомерных изделий сложной (неплоской) формы из TiNi-сплава для медицины достаточно нанесения на их поверхность покрытий на основе титана и тантала толщиной менее $2 \mu \mathrm{m}$, в которых концентрация тантала не превышает 40 at.\%; (ii) данные покрытия можно использовать для повышения рентгеноконтрастности большинства тонкомерных хирургических имплантатов и медицинских устройств сложной формы (фиксаторы, захваты, зажимы, металлические сетки для кардиохирургии, ортопедии и офтальмологии, внутрисосудистые стенты, ловушки и пр.). При этом в отличие от стандартного метода визуализации миниатюрного устройства с помощью меток из контрастных материалов, прикрепляемых лишь на верхней и нижней границах имплантата, данный способ позволяет визуализировать контуры всего изделия, что обеспечивает возможность его точного позиционирования, правильного раскрытия и контроля за формой и целостностью как в процессе операции, так и в послеоперационный период. 
Основные выводы исследований:

1. Согласно теоретической оценке, концентрация тантала 30-40 at.\% в сплаве Тi-Та приводит к увеличению коэффициента поглощения сплава в $\sim 10$ раз по сравнению со значением для TiNi-сплава или чистого титана.

2. Показано, что покрытия $\mathrm{Ti}_{60-70} \mathrm{Ta}_{40-30}$ толщиной $1-2 \mu \mathrm{m}$ на поверхности проволоки TiNi $(\varnothing 152 \mu \mathrm{m})$ повышают ее рентгеноконтрастность при режимах рентгеновских съемок, используемых на практике, в $~ 2$ раза, что сопоставимо с рентгеноконтрастностью этой проволоки, имеющей покрытие из тантала толщиной $2 \mu \mathrm{m}$.

3. На основании представленных данных можно дать рекомендацию по оптимальному режиму рентгеновской съемки, который, с одной стороны, позволит получить достаточную контрастность материала, с другой стороны, использовать более щадящий для пациента режим при проведении операции.

\section{Благодарности}

Авторы выражают благодарность сотрудникам ИСЭ CO РАН к.ф.-м.н. А.Б. Маркову и Е.В. Яковлеву за модификацию поверхности TiNi сплавов.

\section{Финансирование работы}

Исследования проведены в рамках Программы фундаментальных научных исследований государственных академий наук на 2013-2020 г., научное направление III.23 и VII.65.

\section{Конфликт интересов}

Авторы заявляют, что у них нет конфликта интересов.

\section{Список литературы}

[1] Cheng Y., Cai W., Li H., Zheng Y. // J. Mater. Sci. 2006. Vol. 41. P. 4961-4964.

[2] Park C., Kim S., Kim H.-E., Jang T.-S. // Surf. Coat. Technol. 2016. Vol. 305. P. 139-145.

[3] Zhou Y., Li M., Cheng Y., Zheng Y., Xi T., Wei S. // Surf. Coat. Technol. 2013. Vol. 228. P. S2-S6.

[4] Balla V.K., Bose S., Davies N.M., Bandyopadhyay A. // Biological and Biomedical Materials. 2010. Vol. 62. N 7. P. 61-64.

[5] Oh M.H., Lee N., Kim H., Park S.P., Piao Y., Lee J., Jun S.W., Moon W.K., Choi S.H., Hyeon T. // J. Am. Chem. Soc. 2011. Vol. 133. P. 5508-5515.

[6] Марчук М.С., Мутылина И.Н. // Вологдинские чтения. 2010. № 78. C. $67-69$.

[7] Medical Coatings and Deposition Technologies / Eds: D. Glocker, S. Ranade. Shrivener Publish., Willey, NJ, 2016. P. 115-130. DOI: $10.1002 / 9781119308713 . c h 4$

[8] Физические величины. Справочник / Под ред. И.С. Григорьева, Е.З. Мейлихова. М.: Энергоиздат, 1991. 1232 с.
[9] Materials Properties Handbook: Titanium Alloys / Eds: G. Welsch, R. Boyer, E.W. Collings. ASM International. 1994. P. $1035-1048$.

[10] Коротаев А.Д., Литовченко И.Ю., Овчинников С.В. // Физ. мезомех. 2018. Т. 21. № 5. С. 82-89.

[11] Buenconsejo P.J.S., Kim H.Y., Hosoda H., Miyazaki S. // Acta Mater. 2009. Vol. 57. N 4. P. 1068-1077. DOI: $10.1016 /$ j.actamat.2008.10.041

[12] Zhou Y.L., Niinomi M., Akahori T. // Mater. Sci. Engineer. A. 2004. Vol. 371. P. 283-290.

[13] Motemani Y., Kadletz P.M., Maier B., Rynko R., Somsen C., Paulsen A., Frenzel J., Schmahl W.W., Eggeler G., Ludwig A. // Adv. Eng. Mater. 2015. Vol. 17. P. 1425-1433. DOI: $10.1002 /$ adem.201400576

[14] Motemani Y., Buenconsejo P.J.S., Ludwig A. // Shape Mem. Superelasticity. 2015. Vol. 1. P. 450-459. DOI: $10.1007 / \mathrm{s} 40830-015-0041-0$

[15] ГОСТ P 56924-2016 